\title{
Superficial temporal artery-superior cerebellar artery bypass and trapping of a fusiform aneurysm using intradural anterior petrosectomy: technical case report and anatomical study
}

\author{
Soichi Oya, MD, PhD, ${ }^{1}$ Masahiro Indo, MD, PhD, ${ }^{1}$ Masabumi Nagashima, MD, $\mathrm{PhD},{ }^{2}$ and \\ Toru Matsui, MD, DMSc ${ }^{1}$ \\ 1Department of Neurosurgery, Saitama Medical Center; and 'Department of Anatomy, Saitama Medical University, Saitama, \\ Japan
}

\begin{abstract}
Aneurysms at the distal portion of the superior cerebellar artery (SCA) are very rare. Because of the deep location and a propensity for nonsaccular morphology, aneurysm trapping or endovascular occlusion of the parent artery are the usual treatment options, which are associated with varying risks of ischemic complications. The authors report on a 60 -year-old woman who had a 3.5-mm unruptured aneurysm in the lateral pontomesencephalic segment of the SCA with a significant interval growth to $8 \mathrm{~mm}$. Direct surgical intervention comprising trapping of the aneurysm through a subtemporal approach and intradural anterior petrosectomy combined with revascularization of the distal SCA using the superficial temporal artery (STA) was performed. This approach provided sufficient space for the bypass instruments to be introduced into the deep surgical field at a more favorable angle to enhance microscopic visualization of the anastomosis with minimal retraction of the temporal lobe. The patient was discharged with no neurological deficit. Preservation of the blood flow in the distal SCA should be attempted to minimize the risk of ischemic injury, particularly when the aneurysms arise in the anterior or lateral segment of the SCA. The authors demonstrate the safety and effectiveness of the intradural anterior petrosectomy for STA-SCA bypass along with a relevant anatomical study.
\end{abstract}

https://thejns.org/doi/abs/10.3171/2018.11.FOCUS18432

KEYWORDS aneurysm; anterior petrosectomy; STA-SCA bypass; superficial temporal artery; superior cerebellar artery

$\mathrm{T}$ HE superficial temporal artery (STA)-superior cerebellar artery (SCA) bypass is an established method for SCA reconstruction, ${ }^{2,14}$ and the preferred recipient sites are the anterior pontomesencephalic segments of the SCA, accessed through a pretemporal approach, or the lateral pontomesencephalic segments of the SCA that are accessed through a subtemporal approach. ${ }^{13}$ However, it is difficult to expose these areas, and retracting the temporal lobe carries a risk of brain swelling or injury to the vein of Labbé. We report a case of a fusiform unruptured aneurysm in the distal SCA that was effectively treated by trapping the aneurysm with an STA-SCA bypass. An intradural anterior petrosectomy was used to expose the SCA, which created a wide operative field and also contributed to the minimization of temporal lobe retraction. The effectiveness of this approach was confirmed in a cadaveric study. Written informed consent was obtained from the patient for publication of this case report with accompanying images and video.

\section{Case Report}

History and Radiological Examinations

A 60-year-old right-handed woman underwent MR im- 

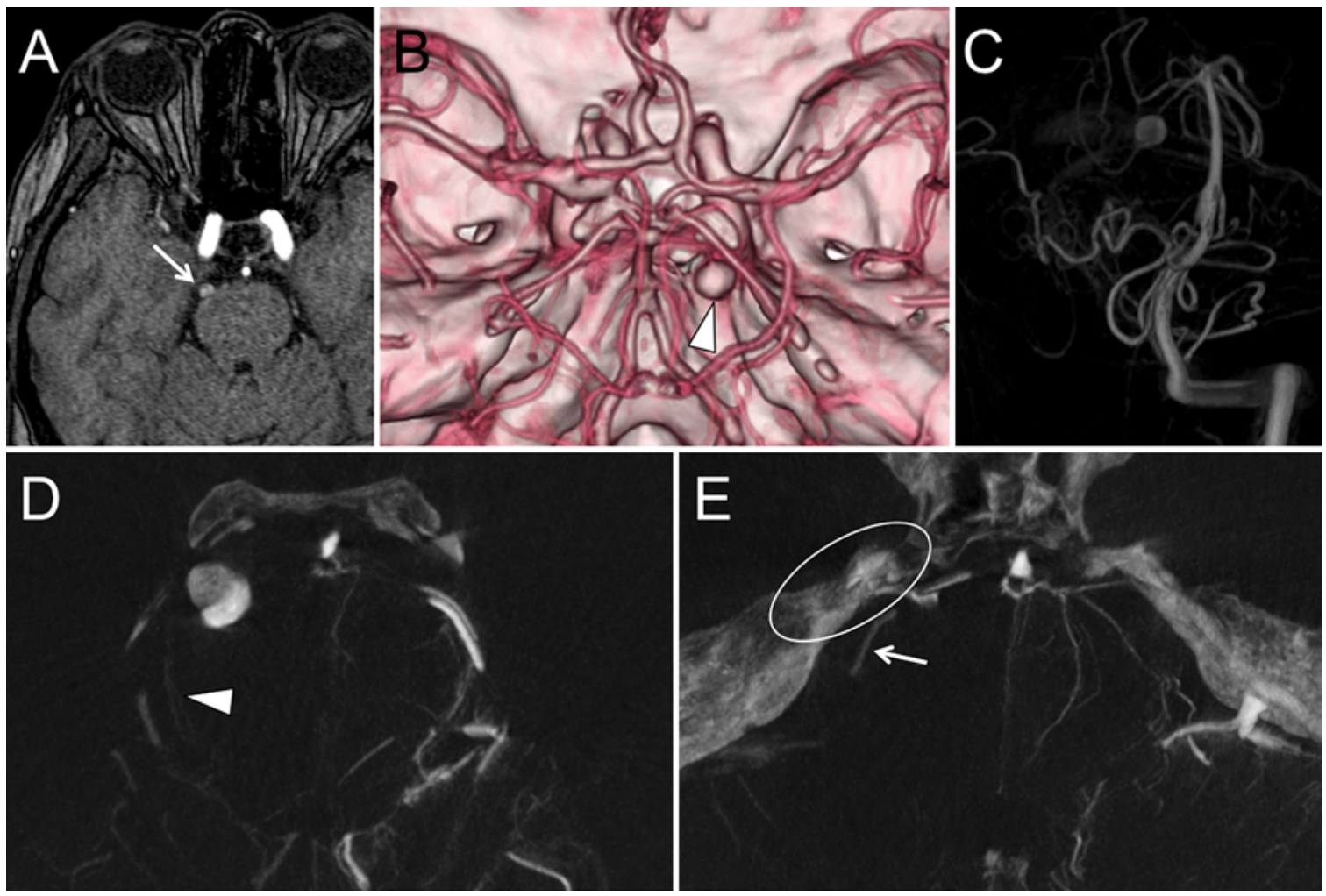

FIG. 1. A: An axial time-of-flight MR angiography sequence showing an incidentally discovered 3.5-mm-diameter aneurysm (arrow) arising in the distal SCA. B: At the 3-year follow-up, 3D-CT angiography revealed that the aneurysm had increased in size (arrowhead) from 3.5 to $5.0 \mathrm{~mm}$, despite which conservative management was continued. C: The angiography study obtained at the 4-year follow-up showed further growth of the aneurysm to $8 \mathrm{~mm}$. The fusiform aneurysm originated at the junction of the anterior and lateral pontomesencephalic segments. D: A preoperative cone-beam CT scan showed perforators (arrowhead) from the SCA penetrating the brainstem. E: A cone-beam CT scan demonstrating the advantage of drilling the petrous apex (oval) to improve access to the distal portion of the SCA (arrow) for constructing the bypass.

aging for chronic headache that initially revealed a 3.5$\mathrm{mm}$ unruptured aneurysm in the distal SCA (Fig. 1A). The third annual follow-up imaging study showed a 5-mm increase in the size of the aneurysm (Fig. 1B). During the next year, the aneurysm showed a significant interval growth to $8 \mathrm{~mm}$ and became fusiform, which originated at the junction of the anterior and lateral pontomesencephalic segments (Fig. 1C). A cone-beam CT scan delineated perforators from the SCA that were penetrating the brainstem, which revealed a risk of ischemic symptoms caused by the SCA occlusion (Fig. 1D). The scan also demonstrated that drilling of the petrous apex would be effective to expose the SCA distal to the aneurysm and to perform a bypass to the SCA (Fig. 1E). The patient preferred to undergo direct surgery comprising the trapping of the aneurysm combined with revascularization of the SCA with the STA.

\section{Surgical Video}

The patient was placed supine with her head rotated $70^{\circ}$ to the left. After a large, curved skin incision was made, the skin flap was anteriorly reflected. The zygomatic arch was sectioned on both ends to reflect the temporal muscle completely downward. After frontotem- poral craniotomy, the temporal bone was drilled to the level of the supramastoid crest. After extradural identification of the petrous apex, the temporal dura mater was sectioned and the temporal lobe was smoothly retracted. We then exposed the petrous apex via an intradural approach by cutting the tentorium and the dura mater of the posterior fossa. The petrous apex was intradurally drilled to expand the operative field. The parietal branch of the STA was anastomosed end-to-side to the SCA at a location distal to the aneurysm. Indocyanine green videoangiography was used to verify the patency of the bypass graft. Last, the aneurysm was trapped using 2 titanium clips (Video 1).

VIDEO 1. The video clip shows a surgical technique for a fusiform aneurysm in the distal SCA treated by bypass and trapping via intradural anterior petrosectomy. Copyright Soichi Oya. Published with permission. Click here to view.

\section{Postoperative Course}

The patient experienced no symptoms after surgery except for temporary trochlear nerve palsy that resolved in 2 weeks. Postoperative MR imaging showed no cerebellar infarction (Fig. 2A). A coronal time-of-flight MR angiography study verified obliteration of the aneurysm and pa- 

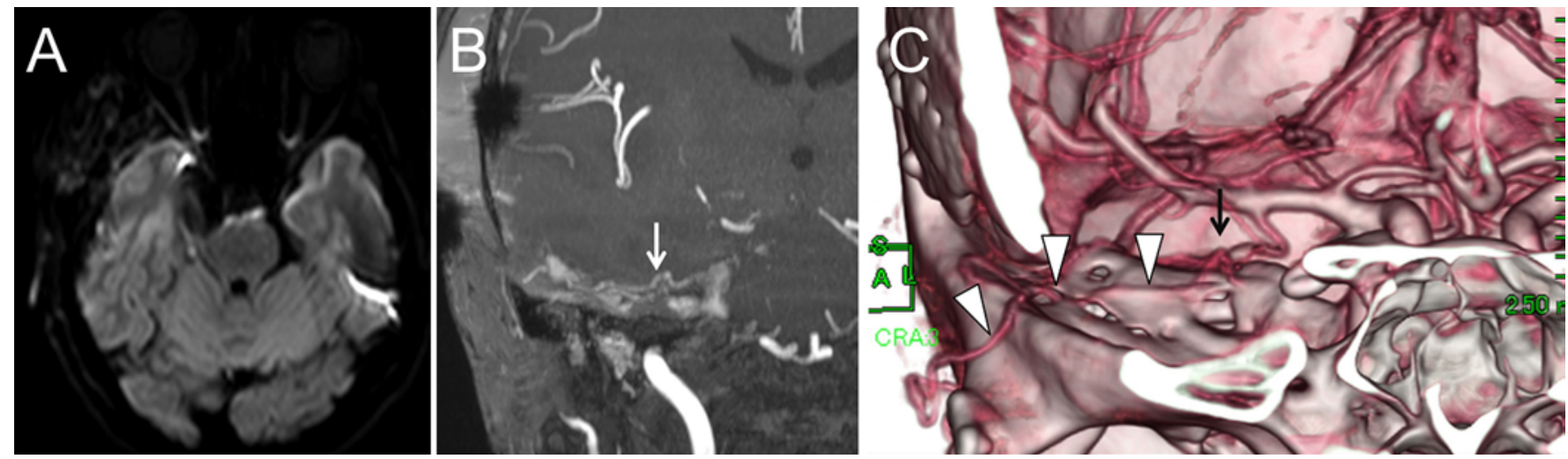

FIG. 2. A: An axial diffusion-weighted MR image obtained 1 day after surgery revealing no ischemic lesion(s) in the territory of the SCA. B: A coronal time-of-flight MR angiography sequence demonstrating patency of the donor artery (arrow) running along the base of the temporal lobe. C: A 3D-CT angiography sequence obtained 1 year after the surgery showing a patent donor STA (arrowheads) and the anastomosis site (black arrow).

tency of the bypass to the distal portion of the SCA (Fig. 2B). The patient was discharged 3 weeks after surgery with no neurological deficits. A coronal slice on 3D-CT angiography obtained 1 year after the surgery demonstrated the patency of the donor artery (Fig. 2C).

\section{Anatomical Study}

We undertook a cadaveric study using 8 sides from 4 formalin-fixed cadaver heads to evaluate the anatomical feasibility, effectiveness, and safety of the intradural anterior petrosectomy. Figure 3 compares the simple left subtemporal approach (Fig. 3A), with that of tentorium sectioning (Fig. 3B), and with that of intradural anterior petrosectomy (Fig. 3C). In Fig. 3, panels D-F are lowmagnification images of panels A-C. Sectioning the tentorium increases exposure of the SCA, which is the standard operative field for STA-SCA bypass (Fig. 3B). The intradural anterior petrosectomy provides significantly more space for anastomosis with the SCA (Fig. 3C).

The actual area in each working field for anastomosis
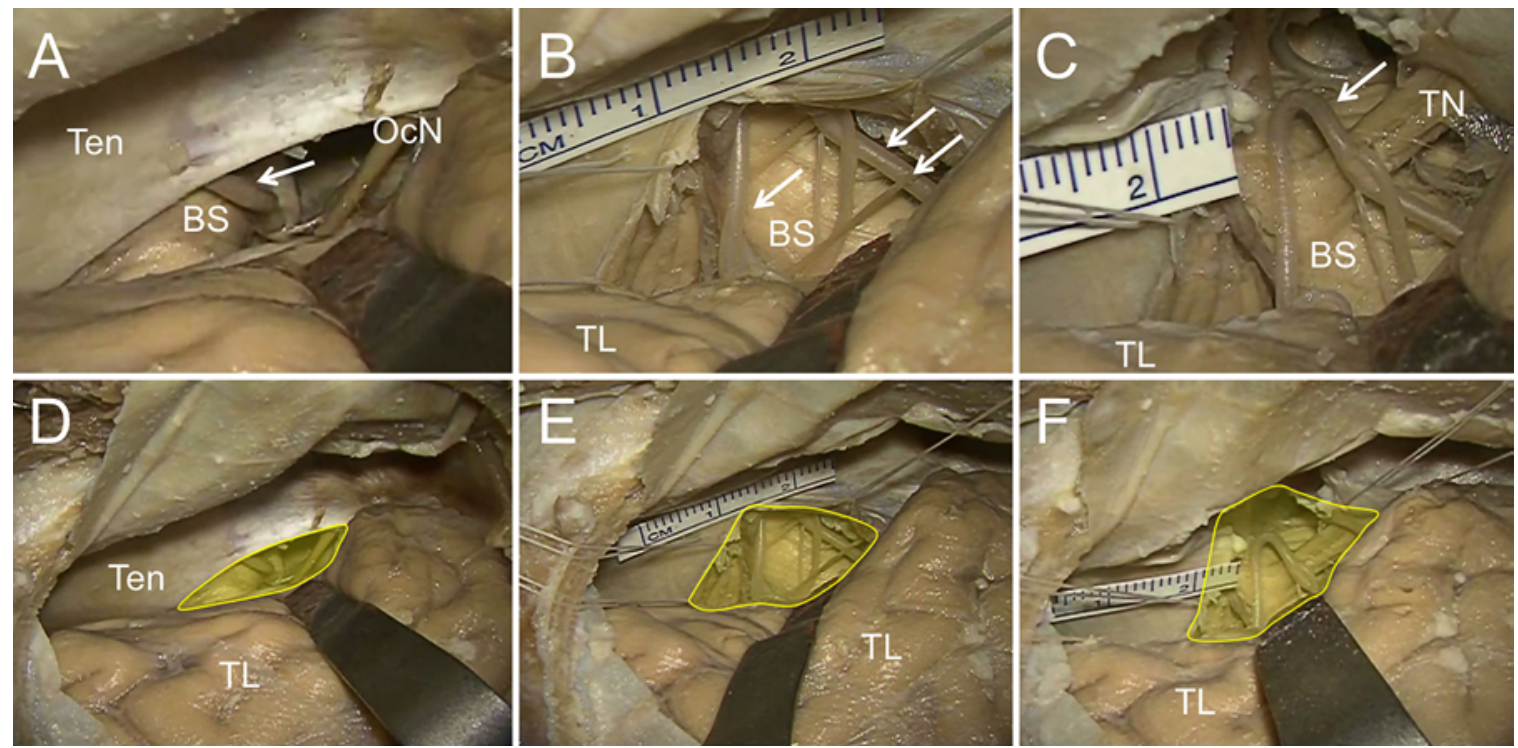

FIG. 3. Cadaveric brain. Comparison among the simple left subtemporal approach (A and D), left subtemporal approach with tentorium sectioning (B and $\mathrm{E}$ ), and the left subtemporal with an intradural anterior petrosectomy ( $C$ and $\mathbf{F})$. $A-C$ and $D-F$ are highand low-magnification images, respectively, of the same area. Only the anterior pontomesencephalic portion of the SCA (arrow) can be seen with the simple subtemporal approach $(A)$. Sectioning the tentorium provides greater exposure of the SCA, which is the standard operative field for constructing a bypass to the SCA (arrows, B). In contrast, the intradural anterior petrosectomy provides significantly more space for anastomosis at the SCA (arrow, C). The yellow areas in D-F indicate the usable surgical field in each approach. Note that the extent of the temporal lobe retraction is much lower when the petrosectomy is added $(F)$. BS = brainstem; OcN = oculomotor nerve; Ten = tentorium; TL = temporal lobe; TN = trigeminal nerve. 
was measured and is indicated as a yellow-shaded area in Fig. 3D-F. The areas of surgical fields were calculated by the equation of ellipse. Surgical fields were larger with a petrosectomy (average $4.6 \mathrm{~cm}^{2}$, range $3.4-5.7 \mathrm{~cm}^{2}$ ) than those without a petrosectomy (average $2.7 \mathrm{~cm}^{2}$, range 2.3 $4.0 \mathrm{~cm}^{2} ; \mathrm{p}=0.001$, 1-tailed paired t-test), and the pressure for temporal lobe retraction was apparently lower on visual inspection with petrosectomy than that without petrosectomy (Fig. 3E and F).

\section{Discussion}

\section{Indication for Revascularization of the Distal SCA}

Treatment options for distal SCA aneurysms include direct clipping, ${ }^{3,7}$ trapping, ${ }^{11}$ wrap/clip reconstruction, ${ }^{8}$ and endovascular coiling/embolization., ${ }^{5,6,13}$ When the shape of the aneurysm is amenable for simple clipping or coiling, patency of the parent artery should be preserved. ${ }^{7}$ However, the incidence of fusiform aneurysms has been previously reported to be as high as $37.5 \%$ among distal cerebellar artery aneurysms. ${ }^{9}$ Several cases of dissecting aneurysms of the distal SCA have also been reported in the literature. ${ }^{8,11}$ Notably, previous reports have mentioned that the risk of ischemic complications appears to be relatively small because of good collateral flow between the SCA and the anterior inferior and posterior inferior cerebellar arteries through the vermis. ${ }^{3,5}$ However, a sufficient collateral flow after SCA occlusion currently cannot be objectively evaluated due to the lack of an appropriate index. ${ }^{1,8,14}$ Ischemic symptoms such as abducent nerve palsy and brainstem dysfunction have been previously reported..$^{1,13}$ If the aneurysms are formed in the cortical segment of the SCA, revascularization would not be necessary. ${ }^{13}$ However, nonsaccular aneurysms are seen more commonly in the proximal portions of the SCA than in the cortical segment, ${ }^{8}$ which emphasizes the importance of revascularization as a therapeutic option for the distal SCA aneurysms. In our opinion, the feasibility of aneurysm trapping and restoration of flow to the portion distal to the aneurysm should be explored to prevent unpredictable ischemia of the brainstem and cerebellum.

\section{Surgical Techniques for STA-SCA Bypass}

The operating field of the subtemporal approach is deep and narrow, thereby introducing difficulty in the bypass, and requires significant temporal lobe retraction. ${ }^{14}$ Additionally, the risk for temporal lobe retraction would further increase while exposing the SCA because the vein of Labbé or the sphenopetrosal vein traverses the surface of the temporal lobe. Based on our anatomical study, intradural drilling of the petrous apex provides a wider working space for bypass than the commonly used subtemporal approach. It also mitigates the necessity for greater retraction of the temporal lobe by providing more space inferior to the SCA. It makes more space for the bypass instruments to be introduced into the deep surgical field at a more favorable angle to enhance microscopic visualization of the anastomosis. Several modifications of the anterior petrosectomy have been reported, $, 10,12,14$ and the approach used by Ichimura et al. ${ }^{4}$ is similar to our approach. We believe that the intradural anterior petrosectomy has advantages over the extradural approach because it provides the right amount of distal SCA exposure to construct a deep bypass with minimal temporal lobe retraction.

\section{Conclusions}

Trapping combined with revascularization of the distal SCA can be safely conducted using the subtemporal intradural anterior petrosectomy approach to expand the room in which to perform the bypass. The results of this case and our cadaveric feasibility study prove that it is important to carefully assess the location of the aneurysm to use it advantageously for choosing appropriate treatment strategies.

\section{References}

1. Alurkar A, Karanam LSP, Nayak S, Oak S: Endovascular management of fusiform superior cerebellar artery aneurysms: a series of three cases with review of literature. J Clin Imaging Sci 2:47, 2012

2. Ausman JI, Diaz FG, de los Reyes RA, Pak H, Patel S, Mehta $\mathrm{B}$, et al: Posterior circulation revascularization. Superficial temporal artery to superior cerebellar artery anastomosis. J Neurosurg 56:766-776, 1982

3. Hayashi N, Kurimoto M, Nagai S, Sato H, Hori S, Endo S: Tentorial incision in a lateral-medial direction with minimal retraction of the temporal lobe in the subtemporal transtentorial approach to the middle tentorial incisural space. Minim Invasive Neurosurg 51:340-344, 2008

4. Ichimura S, Hori S, Hecht N, Czabanka M, Vajkoczy P: Intradural anterior transpetrosal approach. Neurosurg Rev 39:625-631, 2016

5. Jeon JB, Oh SY, Hyun DK, Shim YS: Fusiform superior cerebellar artery aneurysm treated with endovascular treatment. J Cerebrovasc Endovasc Neurosurg 18:276-280, 2016

6. Kang MC, Chae KS, Noh SJ, Choi HG, Ghang CG: Coil embolization of ruptured thrombosed distal superior cerebellar artery aneurysm: a case report. J Cerebrovasc Endovasc Neurosurg 14:243-246, 2012

7. Lyness L, Dehdashti AR: Clipping of distal superior cerebellar artery aneurysm with preservation of the parent vessel: a case report. Br J Neurosurg 26:83-85, 2012

8. Nussbaum ES, Defillo A, Zelensky A, Stoller R, Nussbaum L: Dissecting peripheral superior cerebellar artery aneurysms: report of two cases and review of the literature. Surg Neurol Int 2:69, 2011

9. Rodríguez-Hernández A, Zador Z, Rodríguez-Mena R, Lawton MT: Distal aneurysms of intracranial arteries: application of numerical nomenclature, predilection for cerebellar arteries, and results of surgical management. World Neurosurg 80:103-112, 2013

10. Shibao S, Toda M, Orii M, Fujiwara H, Yoshida K: Various patterns of the middle cerebral vein and preservation of venous drainage during the anterior transpetrosal approach. J Neurosurg 124:432-439, 2016

11. Takeshima Y, Ohmori Y, Nakagawa T, Kaku Y, Kuratsu JI, Yano S: Subarachnoid hemorrhage because of distal superior cerebellar artery dissection in neurofibromatosis type 1. World Neurosurg 105:1036.e15-1036.e19, 2017

12. Tripathi M, Deo RC, Suri A, Srivastav V, Baby B, Kumar S, et al: Quantitative analysis of the Kawase versus the modified Dolenc-Kawase approach for middle cranial fossa lesions with variable anteroposterior extension. J Neurosurg 123:14-22, 2015

13. Yamakawa H, Yoshimura S, Enomoto Y, Nakayama N, Iwama T: Aneurysm arising from the cortical segment of the superior cerebellar artery: a case report and review of the literatures. Surg Neurol 70:421-424, 2008 
14. Zador Z, Lu DC, Arnold CM, Lawton MT: Deep bypasses to the distal posterior circulation: anatomical and clinical comparison of pretemporal and subtemporal approaches. Neurosurgery 66:92-101, 2010

\section{Disclosures}

The authors report no conflict of interest concerning the materials or methods used in this study or the findings specified in this paper.

\section{Author Contributions}

Conception and design: Oya. Acquisition of data: Oya, Indo, Nagashima. Analysis and interpretation of data: Oya. Drafting the article: Oya. Critically revising the article: Indo, Matsui.
Reviewed submitted version of manuscript: all authors. Approved the final version of the manuscript on behalf of all authors: Oya. Statistical analysis: Oya. Administrative/technical/material support: Nagashima, Matsui. Study supervision: Nagashima, Matsui.

\section{Supplemental Information \\ Videos}

Video 1. https://vimeo.com/305501974.

\section{Correspondence}

Soichi Oya: Saitama Medical Center, Saitama Medical University, Saitama, Japan.sooya-tky@umin.ac.jp. 